\title{
UTILIZAÇÃO E APLICAÇÃO DE UM MÉTODO DETERMINÍSTICO ADAPTATIVO OTIMIZADO DEDICADO PARA O AUMENTO DA CONFIABILIDADE E DISPONIBILIDADE DO SERVIÇO AUXILIAR DE SUBESTAÇÕES COM A INSERÇÃO DE FONTE SOLAR FOTOVOLTAICA E EÓLICA
}

\begin{abstract}
Alex de Lima e Silva
Doutorando em Engenharia Elétrica - Eletrônica de Potência, pesquisador, Rio de Janeiro, Brasil Prof.alexdelima@gmail.com

Octávio da Costa Ferrari Engenheiro Eletricista, pesquisador, Rio de Janeiro, Brasil octavio.costa.ferrari@gmail.com

Tallis Amorim Simões Mestrando em Engenharia Elétrica - Sistemas de Energia, pesquisador, Rio de Janeiro, Brasil tallis_as@hotmail.com

Vinicius Coutinho de Oliveira Mestre em Engenharia Elétrica, pesquisador, Rio de Janeiro, Brasil e-mail@e-mail.com
\end{abstract}

\section{RESUMO}

O presente artigo aborda os aspectos técnicos necessários para a utilização e aplicação de um método determinístico adaptativo otimizado para aumentar a confiabilidade de serviço auxiliar de subestações. Considera-se também o uso de geração renovável de energia, no caso solar fotovoltaica e eólica, em termos de usa aplicabilidade para constituir uma fonte adicional de suprimento para este serviço auxiliar. São apresentados os critérios e considerações utilizados no dimensionamento do sistema de controle do sistema de geração solar fotovoltaico e do sistema de geração eólico, que irá otimizar o uso destes recursos para propiciar o aumento da confiabilidade do serviço auxiliar e redução de custos.

Palavras-chave: Serviço Auxiliar; Confiabilidade; Energia Renovável; Geração Solar; Geração Eólica.

\section{UTILIZATION OF A DETERMINISTIC ADAPTABLE OPTIMIZED METHOD FOR RELIABILITY AND AVAILABILITY INCREASE OF AUXILIARY SERVICES OF SUBSTATIONS WITH INSERTION OF SOLAR- PHOTOVOLTAIC AND WIND ENERGY}

\begin{abstract}
This article approaches the aspects related with the utilization and application of a deterministic adaptable optimized method for reliability increase of the auxiliary service of substations. The usage of renewable power sources, specifically solar and wind, is discussed as a means to add an additional power supply for the auxiliary service. The criteria and considerations for the dimensioning and specification of the controller that will optimize the use of each part of the system is shown, which will allow the enhancement of the reliability and availability of the auxiliary system and, also, cost reduction.
\end{abstract}

Keywords: Auxiliary Service; Reliability; Renewable Energy; Solar Energy; Wind energy. 


\section{INTRODUÇÃO}

Os sistemas de elétricos de potência são compostos por três partes principais, a geração, a transmissão e a distribuição. A parcela referente à transmissão tem como função integrar as diferentes instalações geradoras de energia com os centros de carga das unidades consumidores. Dentro dos sistemas voltados para transmissão de energia, estão as subestações de energia, que possui os equipamentos destinados a proteção, medição, manobra e adequação das diversas grandezas elétricas cujo objetivo é auxiliar a na transmissão adequada da energia entre geração e carga.

Um dos componentes de destaque nas subestações e que tem papel crucial no atendimento dos critérios de confiabilidade são os chamados Serviços Auxiliares, responsáveis por fornecer as condições adequadas para o funcionamento de toda a subestação, como por exemplo o suprimento de energia em níveis de tensão adequados ao funcionamento dos sistemas de proteção e controle de todos os equipamentos da subestação.

De acordo com (ANEEL, 2018), ocorreram um total de 1.096 desligamentos forçados no sistema de transmissão em 2017, dos quais uma parte se deu devido à problemas no serviço auxiliar de subestações, tais como a perda da alimentação provida por este aos demais equipamentos. 0 grande número de falhas em serviços auxiliares de subestações e o impacto destas foi o fator motivador para propor um sistema de monitoramento e controle do serviço auxiliar com utilização de fontes de suprimento alternativas para aumentar a confiabilidade.

Tendo em vista a gravidade dos eventos de perda de fonte de energia para o serviço auxiliar, as concessionárias de transmissão ODOYÁ TRANSMISSORA DE ENERGIA S.A., ESPERANZA TRANSMISSORA DE ENERGIA S.A., TRANSMISSORA JOSE MARIA DE MACEDO DE ELETRICIDADE S.A., em parceria com a empresa executora Fox Prime Treinamento e Soluções em Energia iniciaram o projeto de P\&D abordado neste artigo, cuja elaboração e execução atendem à Lei n 9.991/2000, ao manual de P\&D 2012, à REN ANEEL 504/2012 e à REN ANEEL 754/2016.

O projeto de P\&D em desenvolvimento trata-se de um sistema para aumento da confiabilidade do serviço de auxiliar de subestações, que utiliza um método determinístico adaptativo otimizado para integrar o já existente sistema de suprimento do serviço auxiliar à um sistema de geração baseado em energia solar e eólica. Este sistema manterá o serviço auxiliar em operação mesmo na ausência das fontes de suprimento tradicionais, que no caso são a fonte externa proveniente da distribuidora local, a fonte proveniente de transformador abaixado da subestação e o gerador de emergência. O sistema em desenvolvimento funcionará em regime 
permanente uma vez que a geração das fontes solar e eólica são complementares e também não coincidentes, evitando a ocorrência de longos períodos com baixa ou nenhuma geração. Dessa forma, será possível suprir as cargas do serviço auxiliar quando necessário e também reduzir o consumo de energia da subestação.

Até a etapa atual deste projeto, foi realizado o estudo do estado da arte, a avaliação do melhor local para instalação da planta piloto, o dimensionamento dos sistemas de geração solar e eólico e o desenvolvimento do sistema de controle baseado em método determinístico adaptativo otimizado. Esta etapa do projeto tem por objetivo estabelecer todos os parâmetros necessários para instalação do sistema de geração solar e eólico, e a especificação de todos os componentes e lógicas associadas ao sistema de controle baseado em método determinístico adaptativo otimizado.

\section{DESENVOLVIMENTO}

O sistema proposto deverá integrar geradores baseados em fontes de energia diferentes com os equipamentos já presentes em serviço auxiliar de subestações, tais como barramentos CA e CC. Os principais pontos do trabalho estão ilustrados na Figura 1.

Figura 1 - Esquema simplificado do sistema proposto.

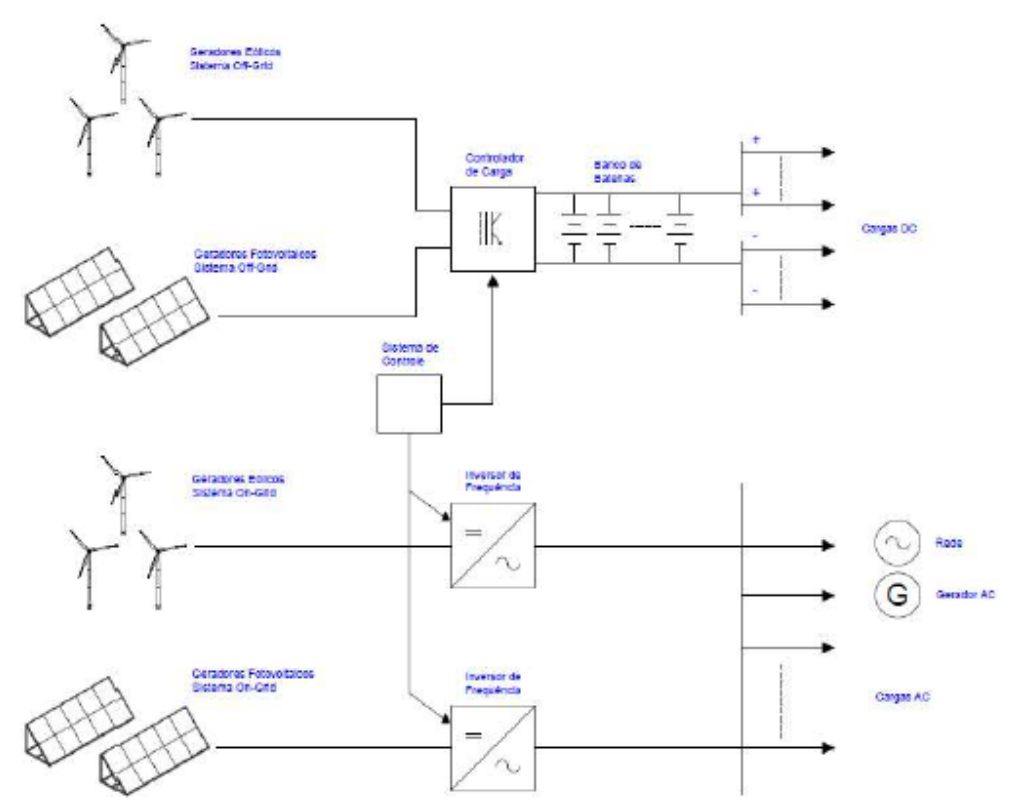

Fonte: (Autoria própria).

Serão descritos os critérios de projeto, bem como a metodologia seguida durante a especificação de cada parte que compõe o projeto.

\subsection{Sistema de Controle}


O objetivo do sistema de controle é otimizar o uso da energia proveniente das fontes renováveis eólica e solar destinadas a abastecer os serviços auxiliares da subestação Morro do Chapéu II. A lógica de controle será implementada em um cartão, cujos três principais componentes são descritos a seguir.

\subsubsection{Microcontrolador}

O hardware do sistema de controle será implementado em um cartão contendo um microcontrolador, o qual será programado com a lógica de controle baseada em um método determinístico adaptativo. Esta lógica tratará de realizar a inferência fuzzy, a partir da aplicação de um conjunto de regras, e de produzir saídas, no caso o chaveamento entre as fontes de abastecimento do serviço auxiliar. Inicialmente, foram apreciados três tipos de microcontrolador:

- PIC-Modelo 16F877A;

- 8051 - Modelo 80C51;

- $\quad$ AVR - Modelo ATMEGA16.

O resultado da avaliação inicial levou a descartar o uso microcontrolador do tipo 8051 devido à indisponibilidade de portas de entrada analógicas no chip, necessárias no uso de conversores A/D externos, maior consumo de energia e menor capacidade de processamento.

Para a presente aplicação, tanto microcontroladores do tipo PIC quanto do tipo AVR são escolhas apropriadas. Para fins da aplicação proposta, será considerado inicialmente o microcontrolador PIC 16F877A como modelo de referências que dispõe de CPU de arquitetura RISC, 10 entradas analógicas para conversores A/D de 8 bits, comunicação serial USART com endereçamento de 9 bits, tensão de alimentação de 2,0 V a 5,5 V, EEPROM com longo tempo de retenção de dados, dentre outras características adequadas à necessidade do presente projeto.

\subsubsection{Interface de Comunicação}

A comunicação entre o cartão do sistema de controle e seus periféricos, tais como controlador de carga e inversores de frequência, se dará através de interfaces seriais padrão RS-485 ou RS-422. Visando a implementar tais interfaces, é necessário a utilização de transceiver que permita a comunicação entre o microcontrolador e a porta serial do periférico. 
O transceiver sugerido para uso é o MAX3140, um integrado com interface UART no terminal do microcontrolador e RS-485/RS-422 no terminal dos periféricos. Esta topologia se adequa à comunicação direta com equipamentos tais como o controlador de cargas híbrido SUNWEY, o inversor de frequência Fronius Primo 5.0-1 e computadores através das respectivas interfaces USB. A Figura 2 ilustra a topologia física do sistema, incluindo a tensão de alimentação e clock externo.

Figura 2 - Topologia física dos elementos de comunicação do sistema de controle.

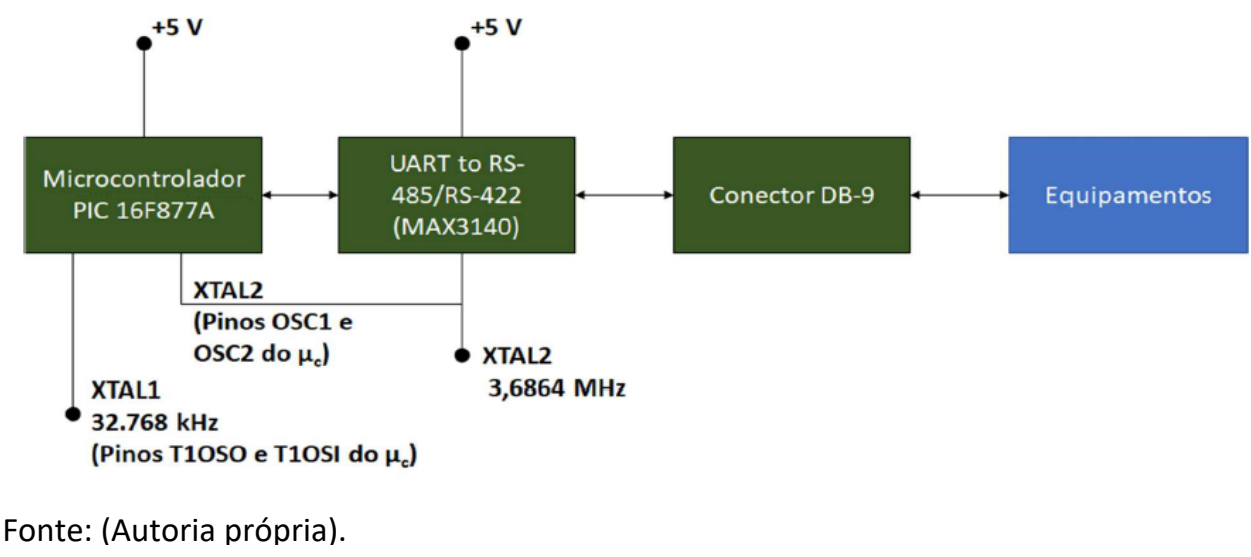

\subsubsection{Fonte de Alimentação}

O cartão contendo o hardware do sistema de controle será instalado em uma subestação de energia de alta tensão, que é um ambiente agressivo sob a ótica de interferências eletromagnéticas. Logo, são necessários alguns cuidados para minimizar os impactos causados por tais interferências na alimentação do circuito.

Para o projeto, é proposto o uso de duas opções de alimentação de circuito: a partir da alimentação da rede em corrente e outra, secundária, alternada uma a partir de uma fonte de $12 \mathrm{~V}$ em corrente contínua conforme ilustrado na Figura 3.

Figura 3 - Digrama de blocos da solução de alimentação do cartão do sistema de controle.

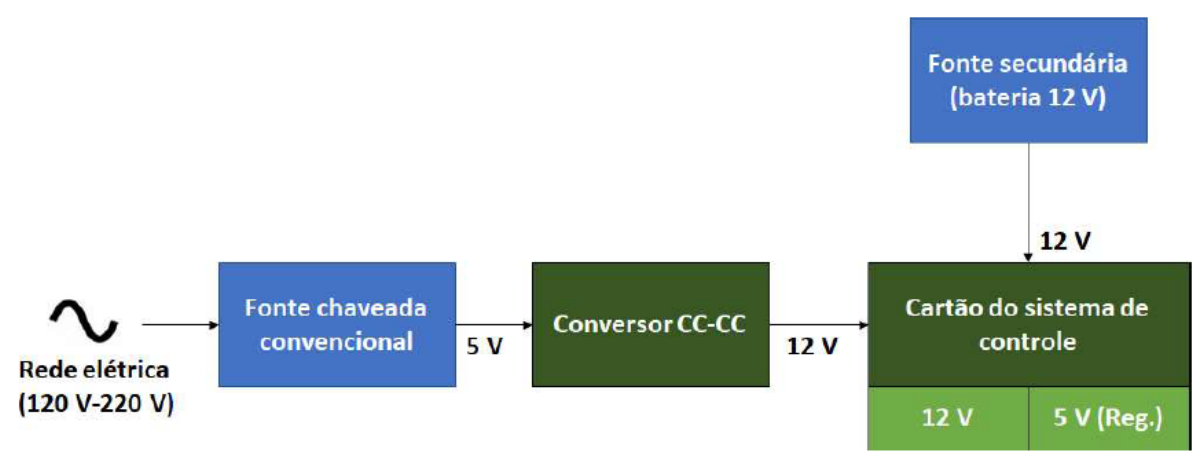

Fonte: (Autoria própria) 
A fonte secundária alimentará diretamente o painel onde fica o cartão do sistema de controle pois é composta por um banco de baterias cuja variação nos valores de tensão estão dentro das especificações do cartão do sistema de controle.

A tensão proveniente da fonte primária, por outro lado, deve ser condicionada antes de ser entregue ao painel. Para retificação do sinal da rede, pode ser utilizada uma fonte chaveada convencional bivolt com saída de $5 \mathrm{~V}$ em corrente contínua. Para evitar interferências induzidas através da rede elétrica, é imprescindível proporcionar o máximo de isolação entre a parte CA e a parte CC do circuito, o que não pode ser garantido por fonte chaveada convencional. Assim, a tensão CC produzida pela fonte chaveada convencional será aplicada a um conversor CC-CC com entrada em $5 \mathrm{~V}$ e saída em $12 \mathrm{~V}$ utilizando o circuito integrado LT1533. Nas partes do circuito em que for necessária tensão menor do que $12 \mathrm{~V}$, será empregada regulação através do circuito integrado LM7805 ou similar.

\subsection{Sistemas de Geração}

O sistema de geração solar fotovoltaico e o sistema de geração eólico serão as fontes de energia utilizadas no projeto em questão, constituindo-se em um sistema híbrido. $O$ uso de mais de uma fonte de geração permite aumentar a confiabilidade frente às incertezas inerentes à incidência de radiação solar e velocidade do vento pois estas apresentam boa complementariedade (CALDAS, 2010). Estas características são observadas no local escolhido para instalação do sistema proposto, que é a Subestação Morro do Chapéu II no estado da Bahia, cuja localização precisa está indicada na Figura 4.

Figura 4 - Localização geográfica da SE Morro do Chapéu II.

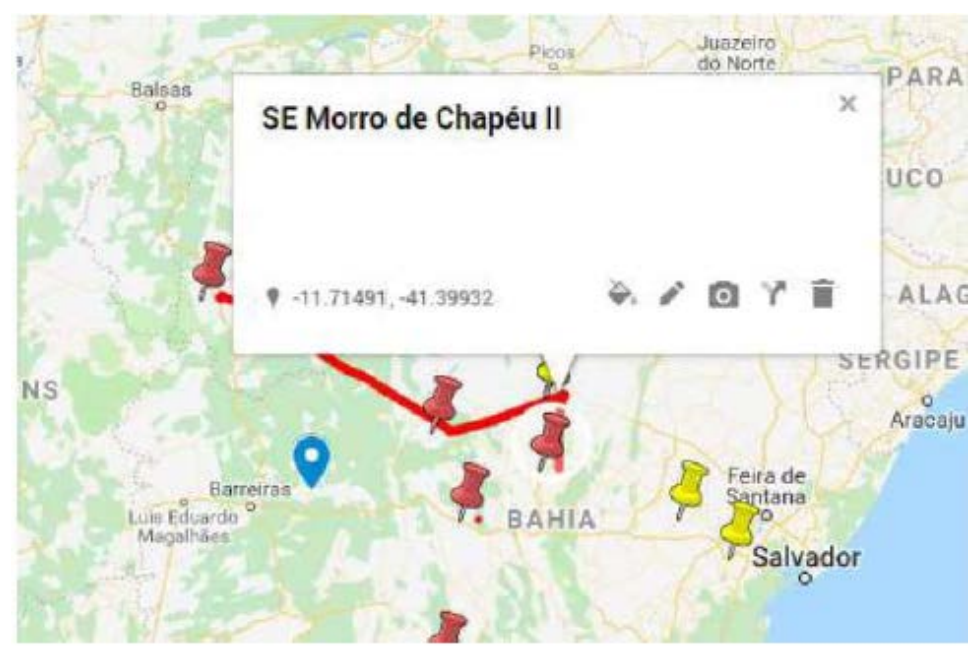

Fonte: (Autoria própria) 
Uma visão simplificada da integração entre as duas fontes está ilustrada na Figura 5.

Figura 5 - Esquema simplificado de um sistema de geração híbrido.

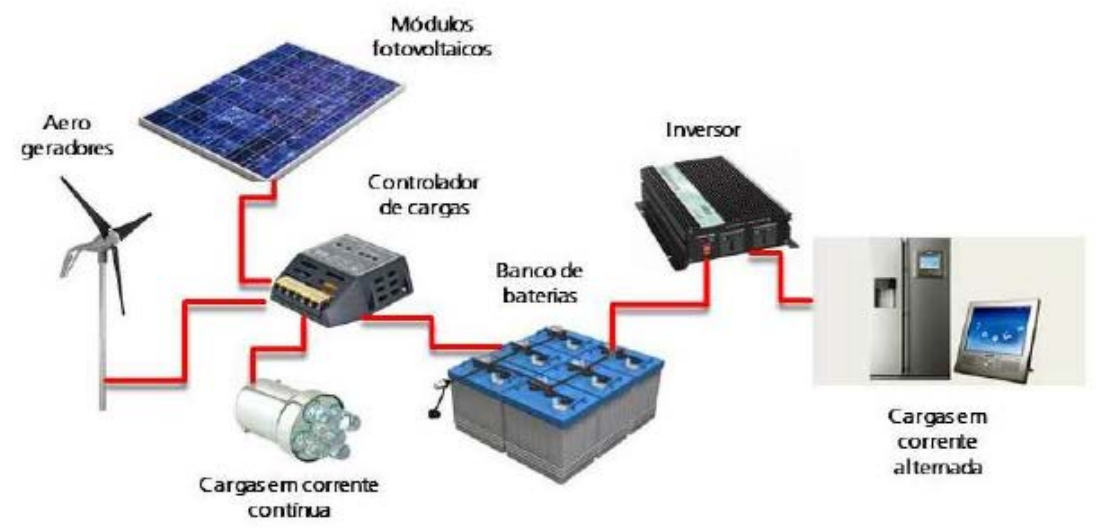

Fonte: (Autoria própria).

Ainda que sejam analisados dentro de um mesmo contexto, o dimensionamento e especificação dos sistemas solar fotovoltaico e eólico podem ser feitos de forma desacoplada. Entretanto, salienta-se que determinados componentes podem ser especificados de forma conjunta para os dois sistemas de geração, permitindo assim uma maior economia tanto financeira quanto de espaço físico graças à integração de mais de uma função em um único equipamento.

\subsubsection{Sistemas de geração solar fotovoltáico}

O Brasil por estar situado em boa parte de seu território na região intertropical, possui uma localização privilegiada para a geração de energia elétrica através da geração solar fotovoltaica. Na Figura 6 é possível observar o grande potencial para geração solar fotovoltaica no Brasil, com destaque para a região Nordeste do país que mantém uma boa média de radiação solar durante todo o ano. 
Figura 6 - Rendimento energético da geração solar fotovoltaica no Brasil.

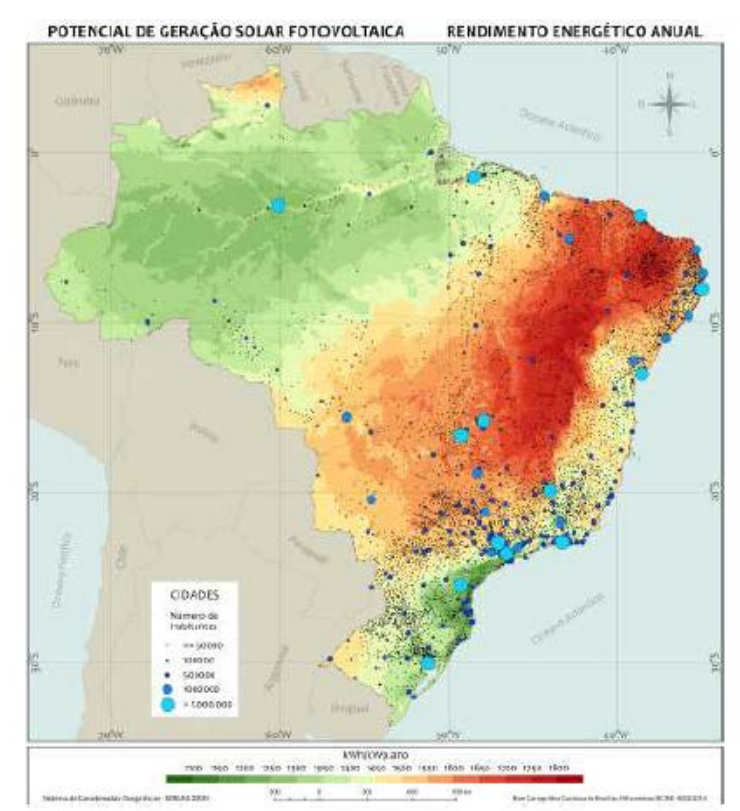

Fonte: (PEREIRA, 2017).

O sistema fotovoltaico é composto principalmente por um conjunto de painéis solares responsáveis por converter energia solar em energia elétrica além de equipamentos destinados a adequar as grandezas elétricas da fonte com os requisitos da carga, tais como inversores de frequência e controladores de carga.

O sistema é dividido em Off-Grid e On-Grid. A parte On-Grid é composta por geradores eólicos e painéis fotovoltaicos interligados a inversores de frequência que alimentam as cargas AC. A parte Off-Grid também é composta por geradores eólicos e painéis fotovoltaicos, sendo interligados por controladores de carga que alimentam um banco de baterias e cargas essenciais em CC.

Um dos fatores mais importantes para definição da viabilidade de se construir um sistema de geração fotovoltaico é a avaliação do potencial de geração solar da região. O objetivo é verificar se o potencial de radiação solar é apropriado para a geração de energia elétrica.

\subsubsection{Dados de Irradiação Solar do Local de Instalação}

Para o estudo do potencial energético solar, foram utilizados dados de satélites geoestacionários. Através de um sistema de coordenadas do local da instalação, foi possível obter os dados climáticos da região próxima da SE Morro do Chapéu II e estabelecer uma área aproximada para as instalações do sistema fotovoltaico. Foram utilizadas as informações de irradiação de 3 
localidades próximas SE Morro do Chapéu II, cujos dados mais relevantes estão resumidos na Tabela 1.

Tabela 1 - Irradiação solar diária média $\left[\mathrm{kWh} / \mathrm{m}^{2}\right.$. dia].

\begin{tabular}{|c|c|c|c|c|c|c|c|c|c|c|c|c|c|c|c|c|}
\hline \multicolumn{3}{|c|}{ Cafarnaum - BA, Brasil } & \multicolumn{14}{|c|}{ I rradiação solar diária média [ kWh/ m².dia] } \\
\hline $\begin{array}{c}\text { Latitude } \\
{\left[{ }^{\circ}\right]}\end{array}$ & $\begin{array}{c}\text { Longitude } \\
{\left[{ }^{\circ}\right]}\end{array}$ & $\begin{array}{c}\text { Distância } \\
\text { [ km] }\end{array}$ & Jan & Fev & Mar & Abr & Mai & Jun & J ul & Ago & Set & Out & Nov & Dez & Média & Delta \\
\hline $11,701^{\circ} \mathrm{S}$ & $41,449^{\circ} \mathrm{O}$ & 0 & 6,4 & 6,16 & 5,91 & 5,02 & 4,5 & 4,31 & 4,5 & 5,22 & 6,1 & 6,35 & 6,22 & 6,43 & 5,58 & 2,13 \\
\hline $11,701^{\circ} \mathrm{S}$ & $41,349^{\circ} \mathrm{O}$ & 10,9 & 6,3 & 6,16 & 5,9 & 5,03 & 4,46 & 4,25 & 4,4 & 5,16 & 6,1 & 6,33 & 6,15 & 6,39 & 5,56 & 2,14 \\
\hline $11,701^{\circ} \mathrm{S}$ & $41,549^{\circ} \mathrm{O}$ & 10,9 & 6,3 & 6,22 & 5,96 & 5,08 & 4,51 & 4,33 & 4,5 & 5,27 & 6,1 & 6,34 & 6,19 & 6,44 & 5,61 & 2,1 \\
\hline
\end{tabular}

Fonte: (Adaptado de CEPEL. www.cresesb.cepel.br, acesso em 22/02/2019).

Conforme premissa proposta por (SILVA, 2013), avaliam-se também os dados de irradiação para o plano inclinado com ângulo igual à latitude do local de instalação, apresentados na Tabela 2.

Tabela 2 - Irradiação solar diária média para plano inclinado[kWh $/ \mathrm{m}^{2}$.dia].

\begin{tabular}{|c|c|c|c|c|c|c|c|c|c|c|c|c|c|c|c|}
\hline \multicolumn{2}{|c|}{ Cafarnaum - BA, Brasil } & \multicolumn{14}{|c|}{ I rradiação solar diária média [ $\mathrm{kWh} / \mathrm{m}^{2}$.dia] } \\
\hline $\begin{array}{c}\text { Longitude } \\
{\left[{ }^{\circ}\right]}\end{array}$ & $\begin{array}{c}\text { Distância } \\
{[\mathrm{km}]}\end{array}$ & Jan & Fev & Mar & Abr & Mai & Jun & Jul & Ago & Set & Out & Nov & Dez & Média & Delta \\
\hline $\begin{array}{c}\text { Plano } \\
\text { Horizontal } \\
\end{array}$ & $0^{\circ} \mathrm{N}$ & 6,36 & 6,16 & 5,91 & 5,02 & 4,5 & 4,3 & 4,47 & 5,2 & 6,08 & 6,35 & 6,22 & 6,43 & 5,58 & 2,13 \\
\hline $\begin{array}{c}\text { Ângulo igual a } \\
\text { latitude }\end{array}$ & $12^{\circ} \mathrm{N}$ & 5,93 & 5,93 & 5,93 & 5,27 & 4,92 & 4,8 & 4,95 & 5,6 & 6,23 & 6,19 & 5,84 & 5,94 & 5,63 & 1,41 \\
\hline $\begin{array}{l}\text { Maior média } \\
\text { anual }\end{array}$ & $9^{\circ} \mathrm{N}$ & 6,05 & 6 & 5,94 & 5,23 & 4,83 & 4,7 & 4,85 & 5,5 & 6,21 & 6,3 & 5,95 & 6,08 & 5,63 & 1,55 \\
\hline $\begin{array}{c}\text { Maior mínimo } \\
\text { mensal }\end{array}$ & $25^{\circ} \mathrm{N}$ & 5,28 & 5,48 & 5,74 & 5,35 & 5,19 & 5,2 & 5,29 & 5,8 & 6,15 & 5,8 & 5,26 & 5,23 & 5,48 & 0,96 \\
\hline
\end{tabular}

Fonte: (Adaptado de CEPEL. www.cresesb.cepel.br, acesso em 22/02/2019).

Definida a área com maior incidência de irradiação, é possível dimensionar os equipamentos do sistema de geração solar-fotovoltaico conforme proposto em (SILVA, 2013). De acordo com esta metodologia, o atendimento da carga off-grid e da carga on-grid será feito por meio de arranjo de um conjunto de painéis fotovoltaicos de $330 \mathrm{~W}$. Um dos modelos de painel fotovoltaico selecionado para a aplicação está detalhado na Tabela 3.

Tabela 3 - Dados do módulo fotovoltaico CS6U 330P.

\begin{tabular}{|c|c|}
\hline Modelo & CS6U 330P \\
\hline Potência nominal máxima & $330 \mathrm{~W}$ \\
\hline Tensão operacional ideal & $37,2 \mathrm{~V}$ \\
\hline Corrente operacional ideal & $8,88 \mathrm{~A}$ \\
\hline Tensão de circuito aberto & $45,6 \mathrm{~V}$ \\
\hline Corrente de curto-circuito & $9,45 \mathrm{~A}$ \\
\hline Eficiência do módulo & $16,97 \%$ \\
\hline Temperatura operacional & $-40{ }^{\circ} \mathrm{C} \sim+85^{\circ} \mathrm{C}$ \\
\hline & $1.000 \mathrm{~V}(\mathrm{IEC})$ ou \\
$1.000 \mathrm{~V}(\mathrm{UL})$
\end{tabular}

Fonte: (Adaptado de CANADIAN SOLAR. www.canadiansolar.com , acesso em 21/02/2019).

No sistema on-grid, é necessário adequar as grandezas elétricas da geração fotovoltaica com as especificações das cargas de CA. O inversor de frequência indicado na Tabela 4 foi selecionado para realizar tal adequação. 
Tabela 4 - Dados do inversor de frequência Fronius Primo 5.0-1.

\begin{tabular}{|r|c|}
\hline \multicolumn{2}{|c|}{ Características de entrada } \\
\hline Voltagem máxima de entrada & $1000 \mathrm{Vcc}$ \\
\hline Faixa de voltagem do MPP & $240 \mathrm{Vcc}$ a $800 \mathrm{Vcc}$ \\
\hline Voltagem mínima de entrada & $80 \mathrm{Vcc}$ \\
\hline Voltagem para inicialização & $80 \mathrm{Vcc}$ \\
\hline Corrente máxima de entrada & $12 \mathrm{~A} / 12 \mathrm{~A}$ \\
\hline Características de saída \\
\hline Potência nominal de saída & $5000 \mathrm{~W}$ \\
\hline Voltagem de saída (faixa) & $180 \mathrm{Vca}$ a $270 \mathrm{Vca}$ \\
\hline Frequência de saída & $60 \mathrm{~Hz}$ \\
\hline Corrente máxima de saída & $21,7 \mathrm{~A}$ \\
\hline
\end{tabular}

Fonte: (Adaptado de FRONIUS. www.fronius.com, acesso em 21/02/2019).

Para o sistema off-grid, deve-se especificar o equipamento responsável por controlar tanto o carregamento do banco de baterias quando o suprimento das cargas CC. A Tabela 5 apresenta os dados de um modelo de controlador de carga capaz de atender às necessidades do projeto.

Tabela 5 - Dados do controlador de carga híbrido SUNWEY SOLAR.

\begin{tabular}{|r|c|}
\hline Potência nominal de energia eólica & $10 \mathrm{~kW}$ \\
\hline Potência nominal de energia solar & $3 \mathrm{~kW}$ \\
\hline Tensão nominal da bateria & $240 \mathrm{~V}$ \\
\hline Over-Tensão de carga & $300 \mathrm{~V}$ (Ajustável) \\
\hline Exibição & LCD com luz de fundo \\
\hline Consumo & $\leq 60 \mathrm{~mA}($ backlight desligado) \\
\hline Coeficiente de temperature-compensation & $-35 \mathrm{mV} /{ }^{\circ} \mathrm{C}\left(25^{\circ} \mathrm{C}\right.$ ref.) \\
\hline Faixa de temperatura ambiente & $-30^{\circ} \mathrm{C}$ a $+55^{\circ} \mathrm{C}$ \\
\hline Faixa de temperatura de armazenamento & $-35^{\circ} \mathrm{C}$ a $+80^{\circ} \mathrm{C}$ \\
\hline Altitude de trabalho & $10-90 \%$ (NC) $)$ \\
\hline Proteção de vidro & $\leq 2000 \mathrm{~m}$ \\
\hline Dados-comunicação (Opcional) & $\mathrm{IP} 55$ \\
\hline RS 485
\end{tabular}

Fonte: (Adaptado de SUNWEY SOLAR. pt.sunwaypv.com, acesso em 21/02/2019).

\subsubsection{Sistema de Geração Eólico}

Uma das premissas deste projeto de P\&D é que o vento seja uma das fontes de energia para o sistema que proverá o aumento da confiabilidade do serviço auxiliar da subestação selecionada. Por este motivo, é necessário que sejam dimensionados os equipamentos que constituirão sistema de geração eólico. Dado que a velocidade do vento apresenta alto grau de incerteza, porém com 
certa sazonalidade, esta pode ser considerada uma variável aleatória cujo valor depende do tempo e, portanto, é um processo estocástico. Assim, as ferramentas de análise de processos estocásticos, tal como o Método de Monte Carlo, são aplicáveis neste caso.

A metodologia comumente aplicada em projetos de sistemas eólicos, via de regra, contempla as etapas de avaliação do vento na região de instalação, que neste trabalho com o Método de Monte Carlo, que, resumidamente, consiste em realizar repetidas amostragens pseudoaleatórias juntamente com análise estatística para inferir resultados. (RAYCHAUDHURI, 2008). A função utilizada para descrever a probabilidade de distribuição do vento é a Distribuição de Weibull dada a sua natureza. A Figura 7 ilustra as etapas seguidas.

Figura 7 - Etapas do projeto do sistema eólico.

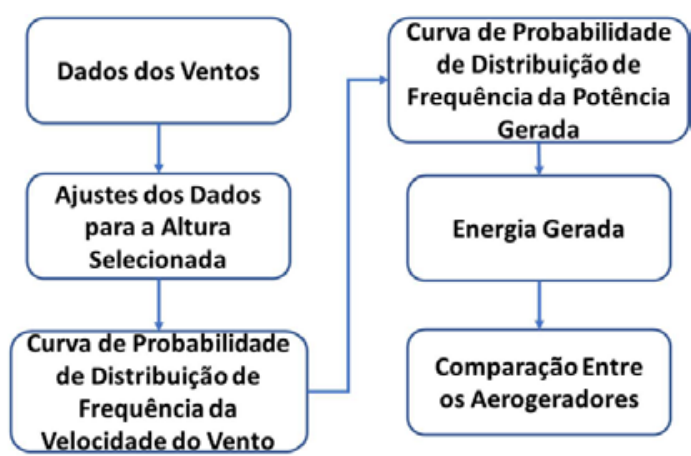

Fonte: (Autoria própria).

\subsubsection{Dados do Vento do Local de Instalação}

Dado que o regime de ventos apresenta um comportamento aleatório e em função do tempo, é possível defini-lo como um Processo Estocástico e, dessa forma, utilizar os métodos probabilísticos para sua análise. Dentre as diversas curvas que descrevem uma distribuição de probabilidade, a que mais se adequa ao comportamento da velocidade dos ventos é a Distribuição de Weibull. A Equação 1 descreve a Distribuição de Weibull.

$$
p(u)=\frac{c}{k} *\left(\frac{u}{c}\right)^{k-1} * e^{-\left(\frac{u}{c}\right)^{k}}
$$

Onde u é a variável sob análise, no caso a velocidade do vento. Restam, então, dois parâmetros para análise da curva de Weibull, o Parâmetro de Forma, k, e o Parâmetro de Escala.

Como será utilizada a Curva de Weibull para descrever a probabilidade de distribuição de frequência da velocidade do vento no local, os dados de vento de entrada utilizados são os fatores c e k da curva de Weibull disponíveis no Atlas do Potencial Eólico Brasileiro (CEPEL, 2013). 
A elaboração do Atlas tem como base o modelo de mesoescala Brams, revisto anualmente (CEPEL, 2013). O sistema responsável por integrar os vários modelos de simulação atmosférica, bases de dados meteorológicos e geográficos e sistemas de armazenamento é o MesoMap, que possui resolução de dados geográficos de $1 \mathrm{~km}$. Utilizando as coordenadas geográficas da localidade selecionada como dado de entrada para o Atlas Eólico do Brasil, são obtidos os dados de vento apresentados na Tabela 6.

Tabela 6 - Dados do vento na localidade escolhida.

\begin{tabular}{|l|c|r|r|r|r|r|}
\hline \multicolumn{7}{|c|}{ Dados de vento a 50 m de altura } \\
\hline \multicolumn{1}{|c|}{ Grandeza } & Unidade & Dez-Fev & Mar-Mai & J un-Ago & Set-Nov & Anual \\
\hline velocidade média do vento & $\mathrm{m} / \mathrm{s}$ & 5,22 & 5,56 & 7,45 & 6,65 & $\mathbf{6 , 2 2}$ \\
\hline fator c & & 5,89 & 6,28 & $\mathbf{8 , 3 3}$ & 7,48 & $\mathbf{7 , 0 1}$ \\
\hline fator k & & 2,37 & 2,27 & 3,13 & 2,63 & $\mathbf{2 , 4 6}$ \\
\hline densidade de potência & W/m2 & 143 & 179 & 348 & 274 & $\mathbf{2 3 6}$ \\
\hline
\end{tabular}

Fonte: (Adaptado de CEPEL, 2013).

Os dados individualizados por trimestre permitem a obtenção de 4 curvas de probabilidade de distribuição, além de uma quinta curva referente aos valores anuais. Entretanto, visto que o objetivo é selecionar um modelo de aerogerador que melhor se adeque à realidade local, serão utilizados dois dos 4 trimestres:

- O trimestre com a menor média de velocidade do vento, para eliminar os aerogeradores com velocidade mínima de geração superior à velocidade mínima do vento;

- O trimestre com a melhor média de velocidade do vento, para selecionar aqueles que terão o melhor aproveitamento eólico nesta fase, evitando vertimento eólico.

O método de Monte Carlo, ou Simulação de Monte Carlo, baseia-se em repetidas amostragens aleatórias e análise estatística para inferir resultados. Na implementação da simulação de Monte Carlo, são utilizados números aleatórios como uma forma de computar uma grandeza que, na realidade, não é aleatória. Uma rotina genérica do Método de Monte Carlo consiste em:

1. Determinar as propriedades estatísticas das possíveis entradas, no caso são os parâmetros da curva de Weibull;

2. Gerar várias séries de possíveis entradas com as mesmas propriedades das descridas no item 1. No caso em estudo, consiste em criar um grande número diferente de cenários de velocidade de vento;

3. Realizar cálculo determinístico com estas séries ou cenários;

4. Analisar estatisticamente os resultados. 
O Método de Monte Carlo, então, consiste em realizar várias amostragens aleatórias a partir de dados estatísticos conhecidos, e o número de amostragens deve ser grande o suficiente para representar satisfatoriamente o comportamento da variável analisada. Por este motivo, foram utilizados os fatores da curva de Weibull para criar 216.000 valores de velocidade de vento para cada trimestre. Uma das formas de se analisar estatisticamente esta massa de dados é utilizando gráficos de Distribuição de Probabilidade de Frequência, que irá permitir avaliar, por exemplo, que o percentual destes 216.000 valores está abaixo de $2 \mathrm{~m} / \mathrm{s}$. Desta forma, todos estes dados podem ser representados por uma única curva.

Conforme ilustrado na Figura 8, o trimestre de junho a agosto, na cor verde, apresentou a velocidade de vento mais favorável para a geração de energia eólica ao passo que o trimestre de dezembro a fevereiro, na cor vermelha, apresentou os piores valores.

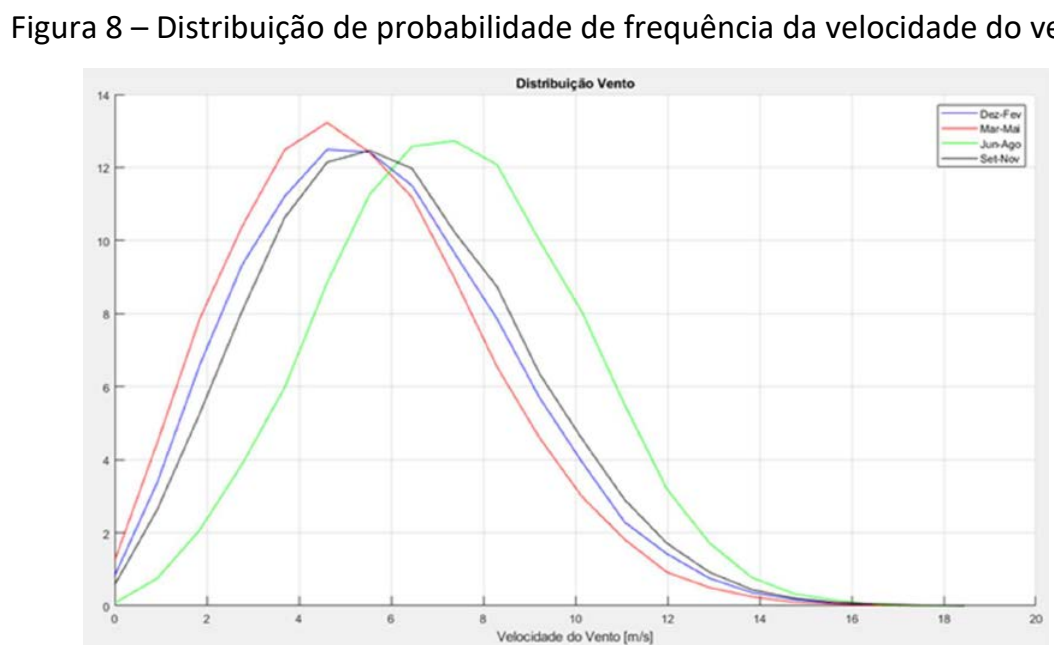

Fonte: (Autoria própria).

Segundo (TRENTINI, 2016), torres de $12 \mathrm{~m}$ costumam possuir a melhor relação custo $\mathrm{x}$ benefício para microgeração e como os valores obtidos do Atlas Eólico são referentes à altura de 50 m, faz-se necessário estimar as velocidades do vento para $12 \mathrm{~m}$. A Equação 2 permite estimar a velocidade do vento à altura das naceles aerogeradores.

$$
V=V o *\left(\frac{\text { Hestimado }}{\text { Hmedido }}\right)^{\alpha}
$$

onde:

- $\mathrm{V}$ : velocidade estimada do vento à altura Hestimado $(\mathrm{m} / \mathrm{s})$;

- Vo: velocidade medida do vento à altura Hmedido $(\mathrm{m} / \mathrm{s})$;

- Hestimado: altura em que se deseja estimar a velocidade do vento (m);

- Hmedido: altura em que se deseja estimar a velocidade do vento (m);

- $\alpha$ : coeficiente de atrito ou rugosidade. 
Os dados de coeficiente de atrito podem ser obtidos na Tabela 7. Considerando que o local de instalação, pode-se utilizar o coeficiente de atrito igual a 0,20.

Tabela 7 - Coeficientes de atrito.

\begin{tabular}{|c|c|}
\hline Característica do terreno & Coficiente de atrito \\
\hline $\begin{array}{c}\text { Calma superfície aquática ou solo } \\
\text { suave }\end{array}$ & 0,10 \\
\hline Grama alta ao nível do solo & 0,15 \\
\hline Arbustos e cercas & 0,20 \\
\hline Áreas rurais com muitas árvores & 0,25 \\
\hline $\begin{array}{c}\text { Pequenas cidades com árvores e } \\
\text { arbustos }\end{array}$ & 0,30 \\
\hline $\begin{array}{c}\text { Grandes cidades com prédios } \\
\text { elevados }\end{array}$ & 0,40 \\
\hline
\end{tabular}

Fonte: (Adaptado de PINTO, 2013).

Os dados de vento foram recalculados e a Figura 9 apresenta as distribuições de probabilidade de frequência da velocidade do vento a $50 \mathrm{~m}$ e a $12 \mathrm{~m}$ para o trimestre com os ventos mais favoráveis.

Figura 9 - Distribuição de probabilidade de frequência do vento à $12 \mathrm{~m}$ e à $50 \mathrm{~m}$ de altura.

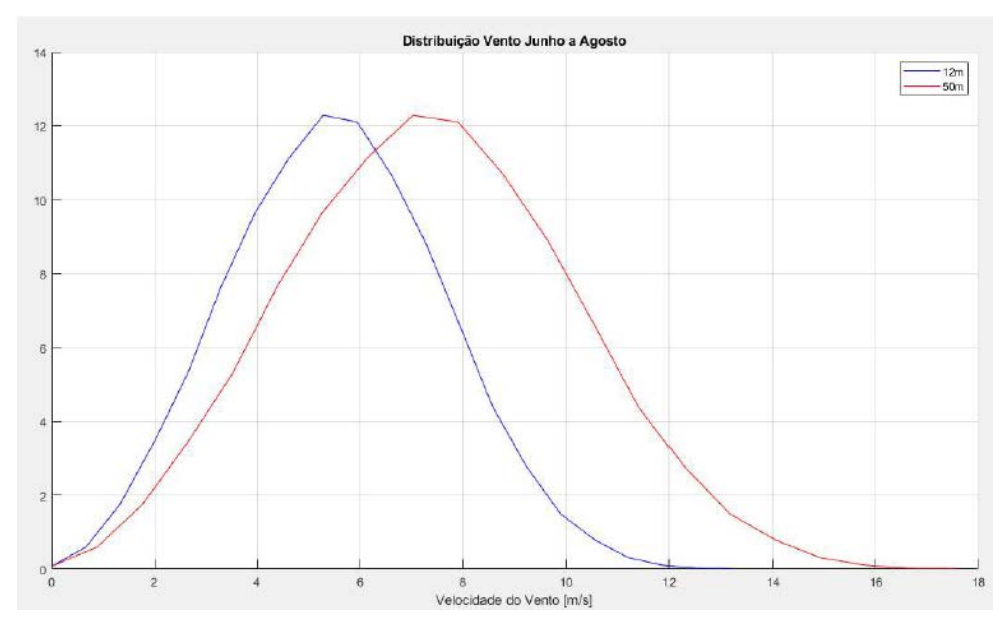

Fonte: (Autoria própria).

\subsubsection{Probabilidade de Distribuição de Potência Gerada}

Um dos aspectos a ser considerados no dimensionamento de um sistema de geração eólico são as características do aerogerador. Os dados obtidos até então permitiram pré-selecionar alguns dos modelos disponíveis no mercado, apresentados nas Tabelas 8, 9 e 10 . 
Tabela 8 -Dados do aerogerador FS-1000.

\begin{tabular}{|c|c|}
\hline Fabricante & WinPower \\
\hline Modelo & FS-1000 \\
\hline Potência Nominal & $1000 \mathrm{~W}$ \\
\hline \multicolumn{2}{|c|}{ Dados do Vento } \\
\hline $\begin{array}{c}\text { Velocidade de } \\
\text { início de carga }\end{array}$ & $3 \mathrm{~m} / \mathrm{s}$ \\
\hline $\begin{array}{c}\text { Velocidade } \\
\text { Nominal }\end{array}$ & $10 \mathrm{~m} / \mathrm{s}$ \\
\hline $\begin{array}{c}\text { Velocidade } \\
\text { Máxima }\end{array}$ & $35 \mathrm{~m} / \mathrm{s}$ \\
\hline
\end{tabular}

Fonte: (Adaptado de WINPOWER. www.chinawinpower.com, acesso em 22/02/2019).

Tabela 9 -Dados do aerogerador iSTA-BREEZE.

\begin{tabular}{|c|c|}
\hline Fabricante & $\begin{array}{c}\text { iSTA- } \\
\text { BREEZE }\end{array}$ \\
\hline Modelo & i-2000 48V \\
\hline Potência Nominal & $2000 \mathrm{~W}$ \\
\hline \multicolumn{2}{|c|}{ Dados do Vento } \\
\hline $\begin{array}{c}\text { Velocidade de início } \\
\text { de carga }\end{array}$ & $2 \mathrm{~m} / \mathrm{s}$ \\
\hline Velocidade Nominal & $12.5 \mathrm{~m} / \mathrm{s}$ \\
\hline Velocidade Máxima & $60 \mathrm{~m} / \mathrm{s}$ \\
\hline
\end{tabular}

Fonte: (Adaptado de iSTA-BREEZE. www.istabreeze.com, acesso em 22/02/2019).

Tabela 10 -Dados do aerogerador Skystream 3.7.

\begin{tabular}{|c|c|}
\hline Fabricante & $\begin{array}{l}\text { Wind } \\
\text { Power }\end{array}$ \\
\hline Modelo & $\begin{array}{c}\text { Skystream } \\
3.7\end{array}$ \\
\hline Potência Nominal & $2400 W$ \\
\hline \multicolumn{2}{|l|}{ Dados do Vento } \\
\hline $\begin{array}{c}\text { Velocidade de início de } \\
\text { carga }\end{array}$ & $3.5 \mathrm{~m} / \mathrm{s}$ \\
\hline Velocidade Nominal & $13 \mathrm{~m} / \mathrm{s}$ \\
\hline Velocidade Máxima & $25 \mathrm{~m} / \mathrm{s}$ \\
\hline
\end{tabular}

Fonte: (Adaptado de WIND POWER. www.windpowerenergiaeolica.com, acesso em 22/02/2019).

Com as curvas das velocidades adaptadas para a altura em que os aerogeradores serão instalados, pode-se utilizar os dados de relação Velocidade do Vento x Potência de cada gerador para obter a curva de probabilidade de distribuição de geração de cada um dos modelos selecionados. Com a curva de velocidade do vento $\mathrm{x}$ potência gerada e as curvas de probabilidade de distribuição de frequência da velocidade do vento obtidas, é possível gerar a curva de probabilidade de distribuição de frequência de geração para cada modelo de aerogerador. As curvas resultantes para o aerogerador i-2000 para dois trimestres do ano estão ilustradas nas Figuras 10 e 11. 
Figura 10 - Distribuição de probabilidade de frequência a potência gerada pelo modelo i-2000 no trimestre mais favorável do ano.

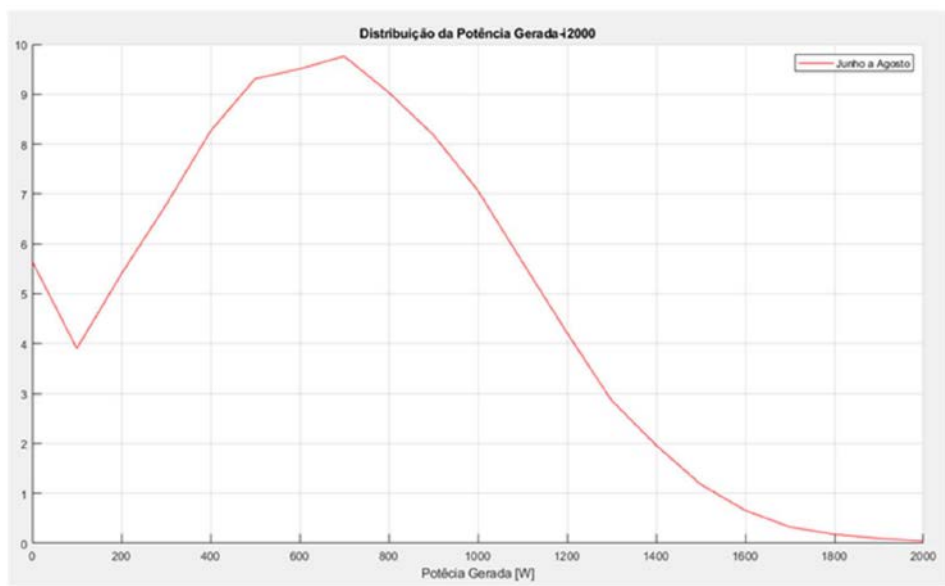

Fonte: (Autoria própria).

Figura 11 - Distribuição de probabilidade de frequência a potência gerada pelo modelo i-2000 no trimestre menos favorável do ano.

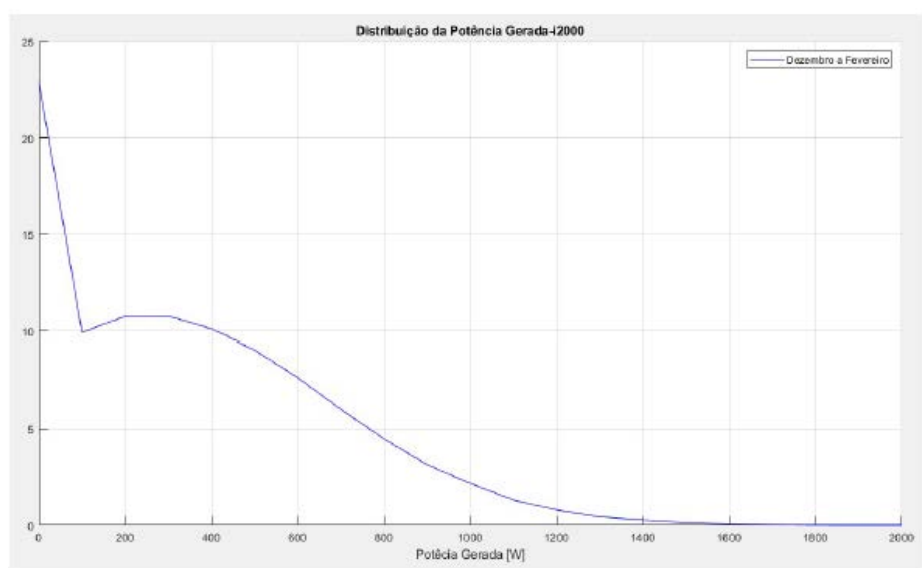

Fonte: (Autoria própria).

\subsubsection{Resultados}

A análise dos casos mostrou que todos os aerogeradores são capazes de gerar sem risco de vertimento eólico até mesmo no trimestre com os maiores valores de velocidade do vento. Entretanto, as baixas velocidades do vento nos meses de dezembro a fevereiro são, eventualmente, insuficientes para a geração eólica, o que pode ser observado na imagem da probabilidade de distribuição de geração do gerador FS-1000. Nota-se, na Figura 12, que o FS-1000 permaneceu sem gerar energia elétrica em $40 \%$ dos 216.000 cenários gerados. 
Figura 12 - Distribuição de probabilidade de frequência a potência gerada pelo modelo FS-1000 no trimestre menos favorável do ano.

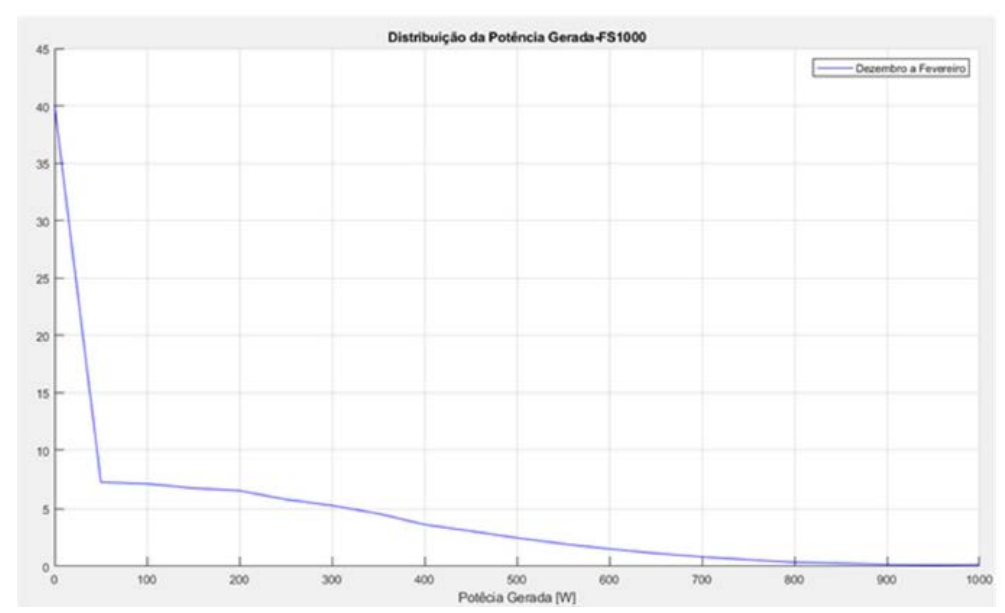

Fonte: (Autoria própria).

Para se obter o valor da energia gerada por cada aerogerador, deve-se calcular o somatório do produto entre os valores de cada ponto da curva de potência do aerogerador pela probabilidade de ocorrência do vento da curva de Weibull, cujo resultado por gerador está na Tabela 11.

Tabela 11 -Expectativa de energia gerada durante o pior trimestre do ano.

\begin{tabular}{|c|c|}
\hline Modelo & Energia Gerada [kWh] \\
\hline FS-1000 & 364.42 \\
\hline i-2000 48V & 825.5 \\
\hline Skystream 3.7 & 881.7 \\
\hline
\end{tabular}

Para permitir uma comparação entre os modelos, calcula-se o fator de capacidade, conforme a Equação 3, de cada um no trimestre mais favorável; assim, pode-se identificar aquele que trará a melhor relação custo x benefício. Os resultados são os da Tabela 12.

$$
\text { Fator de Capacidade }=\frac{\text { Expectativa de Engia Gerada }}{\text { Máxima Energia Gerada Teórica }}
$$

Tabela 12 -Fatores de capacidade de cada modelo de aerogerador.

\begin{tabular}{|c|c|}
\hline Model & Fator de Capacidade \\
\hline FS-1000 & $16.87 \%$ \\
\hline i-2000 48V & $19.11 \%$ \\
\hline Skystream 3.7 & $17.01 \%$ \\
\hline
\end{tabular}

Conforme resultados apresentados acima, o modelo que trará os melhores resultados em termos de geração para a localidade considerada é o modelo Skystream 3.7 e aquele com o melhor fator de capacidade é o i-2000 48V. 
Foram apresentados os principais aspectos e considerações necessárias para a implantação de um método determinístico adaptativo otimizado dedicado para o aumento da confiabilidade e disponibilidade do serviço auxiliar de subestações com a inserção de fonte solar fotovoltaica e eólica. Foram avaliados os critérios de projeto necessários para o correto dimensionamento e integração dos diversos componentes que constituem o sistema final.

O estudo dos sistemas solar e eólico mostrou que a SE Morro do Chapéu II possui boas condições para aproveitar a complementariedade da geração de energia proveniente das duas fontes, sendo este local ideal para aplicação de um protótipo para avaliação da performance do sistema de controle. Foi evidenciado que há uma gama de equipamentos disponíveis no mercado capazes de atender os critérios estabelecidos.

Concluiu-se que a arquitetura proposta para o trabalho deverá adotar microcontrolador da família PIC para implementar um sistema de controle baseado em lógica fuzzy. Desta forma, será possível determinar as condições de operação para elevar a confiabilidade do suprimento do serviço auxiliar da subestação bem como injetar o excedente de energia na rede elétrica, reduzindo o consumo.

Todos principais equipamentos foram dimensionados, restando para as próximas etapas os ensaios em campo para ajuste dos parâmetros do sistema de controle. Tais testes, a serem realizados em etapas futuras do projeto de $P \& D$, permitirão a integração dos sistemas ao serviço auxiliar de qualquer subestação com oferta de energia solar e eólica.

\section{AGRADECIMENTOS}

À ODOYÁ Transmissora De Energia S.A., ESPERANZA Transmissora De Energia S.A. e TRANSMISSORA Jose Maria De Macedo De Eletricidade S.A, que propuseram e financiaram, na forma de um Projeto de P\&D da ANEEL, este trabalho. Ao coordenador do projeto, Prof. Dr. Geraldo Motta Azevedo Junior e ao Engenheiro André Arpon, pelas contribuições fundamentais ao desenvolvimento do trabalho.

\section{REFERÊNCIAS}

ANEEL - Relatório de Análise: desligamentos forçados do Sistema de Transmissão. Brasília, 2018. 
CALDAS, D. M. Estudo do Potencial Eólico e Estimativa de Geração de Energia de um Projeto Eólico na Cidade do Rio de Janeiro utilizando o WindPro e o WAspD. Trabalho de Conclusão de Curso, UFRJ. Rio de Janeiro, 2010.

CEPEL. Atlas do Potencial Eólico Brasileiro - Simulações 2013. Disponível em: http://novoatlas.cepel.br/index.php/atlasonline/ , acesso em 21/02/2019.

PEREIRA, F. A. S.; OLIVEIRA, M. A. S. Curso técnico: Instalador de energia solar fotovoltaica. Porto: Publindústria, 2011.

PINTO, M.O. Fundamentos de energia eólica. Rio de Janeiro, Brasil: LTC, 2013

RAYCHAUDHURI, S. Introduction to Monte Carlo Simulation. Proceedings of the 2008 Winter Simulation Conference, Miami, FL, 2008.

SILVA, A. L.; DEMONT, R. Dimensionamento de um Sistema Fotovoltaico com Armazenamento de Energia. Revista Sodebrás - Volume 8, Número 91, Páginas 10-16, Julho/2013.

TRENTINI, C. Viabilidade Técnico Econômica de Implantação de Aerogeradores em Regiões Brasileiras. Juiz de Fora, MG: UFJF, 2016. 